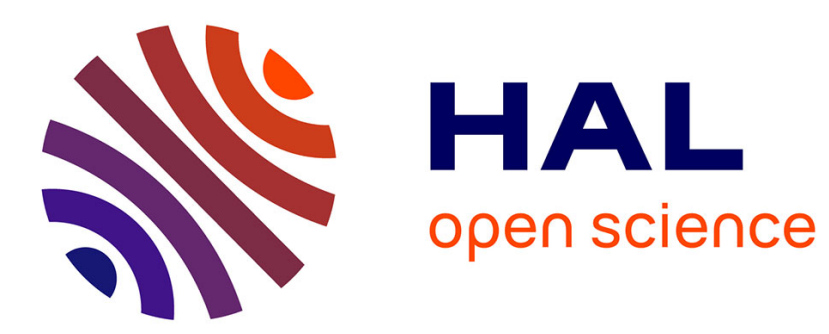

\title{
Le réseau multi-source thermoélastique: une solution au contrôle par ultrasons sans contact
}

\author{
M.-H. Noroy, C. Chenu, D. Royer, M. Fink
}

\section{To cite this version:}

M.-H. Noroy, C. Chenu, D. Royer, M. Fink. Le réseau multi-source thermoélastique: une solution au contrôle par ultrasons sans contact. Journal de Physique IV Proceedings, 1994, 04 (C5), pp.C5-1181C5-1184. 10.1051/jp4:19945260 . jpa-00252950

\section{HAL Id: jpa-00252950 https://hal.science/jpa-00252950}

Submitted on 1 Jan 1994

HAL is a multi-disciplinary open access archive for the deposit and dissemination of scientific research documents, whether they are published or not. The documents may come from teaching and research institutions in France or abroad, or from public or private research centers.
L'archive ouverte pluridisciplinaire HAL, est destinée au dépôt et à la diffusion de documents scientifiques de niveau recherche, publiés ou non, émanant des établissements d'enseignement et de recherche français ou étrangers, des laboratoires publics ou privés. 


\title{
Le réseau multi-source thermoélastique : une solution au contrôle par ultrasons sans contact
}

\author{
M.-H. NOROY, C. CHENU, D. ROYER et M. FINK
}

Laboratoire Ondes et Acoustique, Université Paris VII, URA 1503 du CNRS, ESPCI, 10 rue Vauquelin, 75231 Paris cedex 05, France

\begin{abstract}
Résumé: Un réseau de seize sources thermoélastiques déphasées est utilisé afin d'engendrer des ondes ultrasonores de forte amplitude et de direction réglable. Ce réseau est créé à la surface du solide par un laser Nd:YAG multivoie qui produit seize impulsions indépendantes. En introduisant une loi de retard appropriée entre chaque impulsion laser, on réalise des interférences constructives en tout point du solide. La détection des signaux ultrasonores est assurée soit par un transducteur piézoélectrique soit par une sonde optique. Des défauts en volume ainsi que de fines fentes à la surface du matériau sont détectés respectivement par focalisation des ondes longitudinales et par sommation constructive des ondes de Rayleigh.

Abstract: In order to generate ultrasounds of high amplitude and to achieve beam steering, a 16thermo-elastic sources array is used. This phased array is implemented from a Nd:YAG laser which provides 16 independant optical pulses. Introducing an appropriate time-delay law between each optical pulse, focusing is performed in the solid. The ultrasonic signals are detected either by a piezoelectric transducer or by an optical probe. Bulk defects are detected by longitudinal wave focusing and surface breaking slots by constructive summation of Rayleigh waves.
\end{abstract}

\section{1- INTRODUCTION}

L'utilisation des lasers pulsés est bien adaptée à la génération sans contact d'ondes élastiques dans les solides. Cependant, en régime thermoélastique, l'amplitude des déplacements créés est faible ce qui rend difficile leur détection par une méthode optique. Afin d'améliorer les caractéristiques (directivité, intensité) du faisceau ultrasonore tout en évitant l'ablation du matériau, un réseau de sources thermoélastiques déphasées est utilisé.

Un modèle de source linéique et de réseau de sources déphasées a été développé pour les ondes longitudinales. Les résultats expérimentaux obtenus avec le laser à 16 faisceaux indépendants sont présentés et comparés au modèle. Les ondes longitudinales ainsi focalisées sont utilisées pour détecter des défauts en volume. La même technique peut être mise en oeuvre pour engendrer des déplacements d'ondes de surface de grande amplitude en sommant les ondes de Rayleigh créées par chacune des sources thermoélastiques du réseau. Dans ce cas, la détection de fentes fines localisées à la surface du matériau est possible avec un bon rapport signal sur bruit.

\section{2- ANALYSE}

L'impact d'une impulsion lumineuse sur la surface libre d'un matériau engendre des ondes élastiques de volume et de surface [1]. Pour des densités de puissance correspondant au régime thermoélastique, le déplacement mécanique créé est faible et le diagramme de directivité associé aux ondes longitudinales est large et constitué de deux lobes symétriques centrés aux angles $\alpha= \pm 65^{\circ}$ par rapport à la normale [2].

La source photothermique est une ligne de hauteur $2 b$ et de largeur $2 a(a<<b)$. Dans la direction repérée par l'angle $\alpha$, et à une distance $r$ du centre de la source, le déplacement longitudinal s'exprime comme le produit de convolution de limpulsion laser $\mathrm{q}(\mathrm{t})$ et de la réponse impulsionnelle de diffraction Article published online by EDP Sciences and available at http://dx.doi.org/10.1051/jp4:19945260 
$h(r, \alpha, t)$ de la ligne source.

$$
u_{n}(r, \alpha, t)=q(t) \otimes h(r, \alpha, t)
$$

L'augmentation de la durée du signal acoustique résultant du produit de convolution dépend de la dimension $2 b$. La réponse impulsionnelle de diffraction a été calculée [3] en utilisant les fonctions de directivité appropriées.

Un moyen de réduire la largeur du faisceau acoustique et de contrôler sa direction est de réaliser un réseau de sources photothermiques déphasées [4]. L'instant d'émission de chaque impulsion laser est retardé afin de créer en un point du solide la sommation constructive des impulsions acoustiques.

La figure 1 montre une carte isoniveau (par pas de $2 \mathrm{~dB}$ ) du champ acoustique, engendré par un réseau de seize sources thermoélastiques, pour un point de focalisation situé à une distance de $34 \mathrm{~mm}$ dans la direction $\alpha=64^{\circ}$. Les deux lignes épaisses indiquent la largeur à $-6 \mathrm{~dB}$ du faisceau acoustique $\left(5,3^{\circ}\right.$ au point focal). Ce résultat montre que ce réseau focalise en régime thermoélastique les ondes longitudinales en un faisceau étroit avec une grande profondeur de champ.

La génération d'ondes ultrasonores par impact laser offre aussi la possibilité de créer sans contact des ondes de surface. On peut obtenir la sommation constructive de chacune des impulsions d'onde de Rayleigh en retardant chaque source.

\section{3- PROCEDURE EXPERIMENTALE}

Les expériences ont été menées avec un laser impulsionnel Nd:YAG fabriqué par la société B.M. Industries. Ce laser est constitué de seize têtes indépendantes qui produisent chacune une impulsion de durée inférieure à $30 \mathrm{~ns}$ et de longueur d'onde $1,06 \mu \mathrm{m}$. Chaque faisceau laser est focalisé en une ligne de 8 $\mathrm{mm}$ de haut à une distance de $1 \mathrm{~m}$. Les retards sont contrôlés à la nanoseconde près par ordinateur via une liaison GPIB. On dispose d'un échantillon poli de duralumin de forme hémi-cylindrique de rayon $24 \mathrm{~mm}$ $\left(\mathrm{S}_{1}\right)$. Le deuxième échantillon $\left(\mathrm{S}_{2}\right)$ est un bloc de duralumin $(\mathrm{L}=150 \mathrm{~mm}, l=30 \mathrm{~mm}, \mathrm{~h}=40 \mathrm{~mm})$ et le troisième $\left(\mathrm{S}_{3}\right)$ une plaque de duralumin de $10 \mathrm{~mm}$ d'épaisseur. Un réseau de seize impacts linéiques est créé à la surface du matériau en plaçant celui-ci en avant du plan focal du multi-laser (Fig. 2).

Les ondes longitudinales dans $S_{1}$ et les ondes de Rayleigh à la surface de $S_{3}$ sont détectées respectivement en transmission et en réflexion par un interféromètre. Cet interféromètre, de type MachZehnder hétérodyne, est capable de mesurer des déplacements d'amplitude $0,1 \mathrm{~nm}$ avec une bande passante de $20 \mathrm{MHz}$ [5]. Les ondes élastiques créées dans l'échantillon $\mathrm{S}_{2}$ sont détectées en réflexion avec un transducteur de diamètre $6 \mathrm{~mm}$ (fréquence centrale $6 \mathrm{MHz}$, bande passante à $-6 \mathrm{~dB}$ de $5 \mathrm{MHz}$ ).

\section{4- RESULTATS}

Deux séries d'expériences, l'une avec des ondes de volume longitudinales, l'autre avec des ondes de Rayleigh, sont décrites. L'énergie optique incidente est limitée à $40 \mathrm{~mJ}$ pour chaque voie, évitant ainsi l'ablation du matériau.

\section{4-1- Focalisation des ondes longitudinales et détection de défauts}

Un réseau de seize sources thermoélastiques d'ouverture $24 \mathrm{~mm}$ est utilisé afin de focaliser les ondes ultrasonores à $65^{\circ}$ par rapport à la normale à l'échantillon $S_{1}$. La figure 3 montre le déplacement acoustique transmis à travers l'échantillon et détecté avec la sonde optique. Dans la direction $\alpha=65^{\circ}$, pour laquelle la sommation constructive des seize impulsions acoustiques est obtenue, le maximum d'amplitude du signal acoustique atteint $4 \mathrm{~nm}$. La figure $3(\mathrm{~b})$ correspond au signal acoustique reçu dans la direction $\alpha=-65^{\circ}$ pour laquelle les impulsions acoustiques atteignent le point de détection avec de tels retards qu'elles sont totalement séparées dans le temps.

Un exemple de détection de défaut est présenté en réalisant le balayage sectoriel de l'échantillon $S_{2}$ par le faisceau ultrasonore. Un trou cylindrique de $1 \mathrm{~mm}$ de diamètre dont la génératrice est parallèle aux lignes sources a été percé à $15 \mathrm{~mm}$ de profondeur dans l'échantillon. L'ouverture du réseau est de $25 \mathrm{~mm}$. Le signal acoustique est détecté par le transducteur piézoélectrique. La figure 4 montre le détail d'un B- 
scan obtenu en quelques minutes entre $0^{\circ}$ et $90^{\circ}$ avec un pas angulaire de $0.5^{\circ}$.

Le signal acoustique diffracté par le défaut a une amplitude maximale pour $65^{\circ}$ et une largeur à $-6 \mathrm{~dB}$ de $9^{\circ}$. Ce résultat montre que le réseau de sources thermoélastiques est capable de créer des ondes acoustiques d'amplitude suffisante pour détecter des défauts par balayage sectoriel de l'échantillon en régime thermoélastique.

\section{4-2- Sommation constructive des ondes de Rayleigh et détection de fentes étroites}

Un réseau d'ouverture $28 \mathrm{~mm}$ est utilisé pour engendrer des ondes de Rayleigh à la surface de l'échantillon $\mathrm{S}_{3}$. Le faisceau laser de la sonde optique est focalisé à $26 \mathrm{~mm}$ de la ligne source la plus proche. Un train de 16 impulsions d'ondes de Rayleigh est engendré lorsque les impulsions laser sont toutes émises en même temps (Fig. 5a). L'introduction de retards convenables permet la sommation constructive des déplacements mécaniques, conduisant à une onde de Rayleigh unique de très forte amplitude : $50 \mathrm{~nm}$ crête à crête (Fig. $5 b)$. Etant donnée la somme $(53 \mathrm{~nm})$ des amplitudes (crête à crête) des signaux élémentaires de la figure 5a, la sommation constructive est réalisée avec un bon rendement (94\%). Les premiers signaux observés sur la figure $5 \mathrm{~b}$ sont d'origine électromagnétique, ils correspondent aux déclenchements successifs des seize impulsions laser.

Afin de mettre en évidence des défauts superficiels, l'échantillon $S_{3}$ a été préparé avec une série de fentes de même largeur $(0,2 \mathrm{~mm})$ et de différentes profondeurs $(\mathrm{h}=1 \mathrm{~mm}, 0,4 \mathrm{~mm}$ et $0,1 \mathrm{~mm})$. La configuration réseau-sonde précédente est conservée et est placée d'un même côté de la série de fentes. Le faisceau sonde est focalisé à $9 \mathrm{~mm}$ de chaque fente étudiée. Comme le montre la figure 6 , l'onde de Rayleigh incidente résultant de la sommation constructive est détectée en premier, suivie par le signal réfléchi par la fente. L'amplitude du premier pic négatif de l'onde réfléchie est relativement importante, respectivement $44 \%, 38 \%$ et $17 \%$ de celle de l'onde incidente. Une fente de $0,1 \mathrm{~mm}$ de profondeur est facilement détectable en régime thermoélastique grâce au principe de la sommation constructive du fait de la forte amélioration du rapport signal sur bruit.

\section{5- CONCLUSION}

Un réseau de sources linéiques déphasées a été créé à la surface d'un solide en utilisant un laser YAG à seize faisceaux. Les expériences réalisées en régime thermoélastique ont démontré qu'un faisceau ultrasonore étroit de direction variable pouvait être engendré dans le volume du solide. Ce faisceau peut être dirigé entre $25^{\circ}$ et $75^{\circ}$ en changeant les retards entre chaque impulsion optique. Les résultats expérimentaux sont en bon accord avec les simulations qui tiennent compte à la fois des effets de diffraction introduits par la source linéique et des diagrammes de directivité. L'augmentation sensible $(\times 15)$ de l'amplitude des ondes longitudinales rend possible la détection de défauts en volume par balayage électronique du faisceau ultrasonore. Le réseau de sources thermoélastiques engendre aussi par sommation des ondes de Rayleigh de grande amplitude à la surface de l'échantillon, favorisant ainsi la détection en régime thermoélastique de fentes étroites.

\section{REFERENCES}

[1] D. A. Hutchins, "Mechanisms of pulsed photoacoustic generation", Can. J. Phys., 64, 1247 (1986).

[2] L. R. F. Rose, "Point-source representation for laser-generated ultrasound", J. Acous. Soc. Am., 75 (3), 723-732 (1984).

[3] M.-H. Noroy, D. Royer and M. Fink, "The laser generated ultrasonic phased array: Analysis and experiments", J. Acous. Soc. Am., 94 (4), 1934-1943 (1993).

[4] J. A. Vogel, A. J. A. Bruinsma and A. J. Berkhout, "Beamsteering of laser generated ultrasound", Proc. Ultrason. Int. 87 Conf., 141-152 (1987).

[5] D. Royer and E. Dieulesaint, "Optical probing of the mechanical impulse response of a transducer", Appl. Phys. Lett., 49, 1056-1058 (1986). 


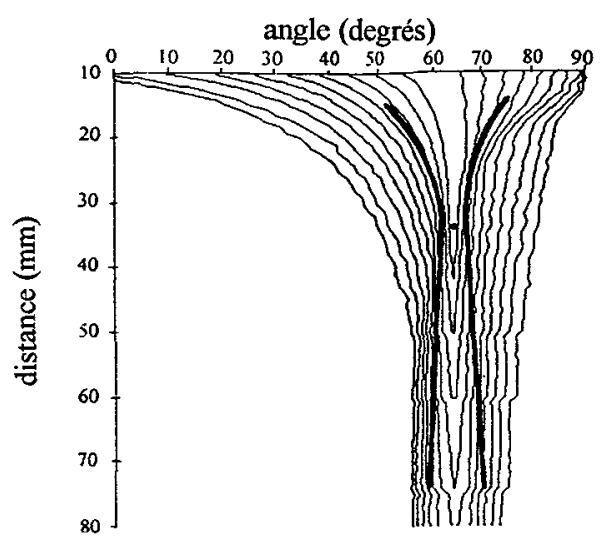

Fig. 1. Courbes isoniveaux (par pas de $2 d B$ ) $d u$ champ acoustique pour $10 \mathrm{~mm}<r<80 \mathrm{~mm}$. Le point focal est situé à $r=34 \mathrm{~mm}$ et $\alpha=64^{\circ}$.

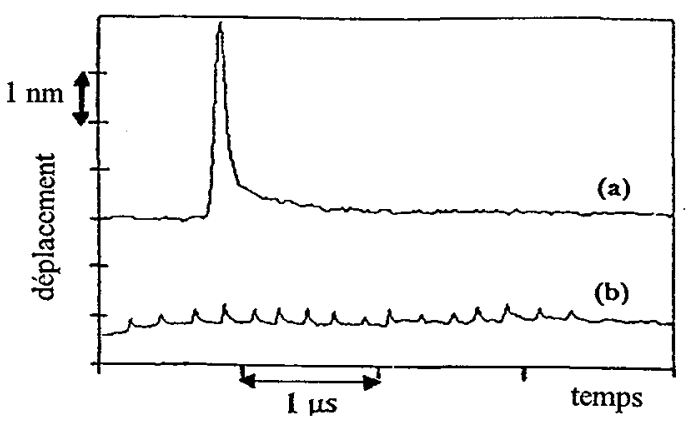

Fig. 3. Déplacements acoustiques longitudinaux détectés avec la sonde optique, (a) au point focal pour $\alpha=65^{\circ}$. (b) dans la direction symétrique ( $\alpha$ $=-65^{\circ}$ ) pour laquelle il n'y a pas de focalisation.

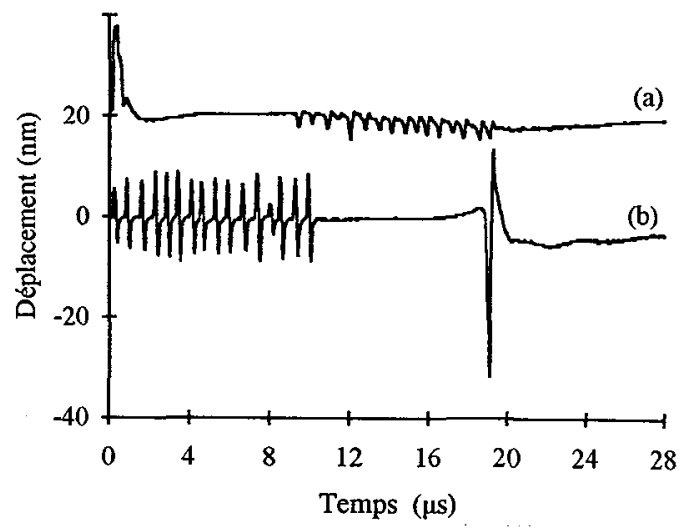

Fig. 5. Sommation constructive des ondes de Rayleigh. Le déplacement acoustique est détecté par la sonde optique. (a) Sans aucun retard. (b) Au même point d'observation avec des retards convenables entre chaque impulsion optique.

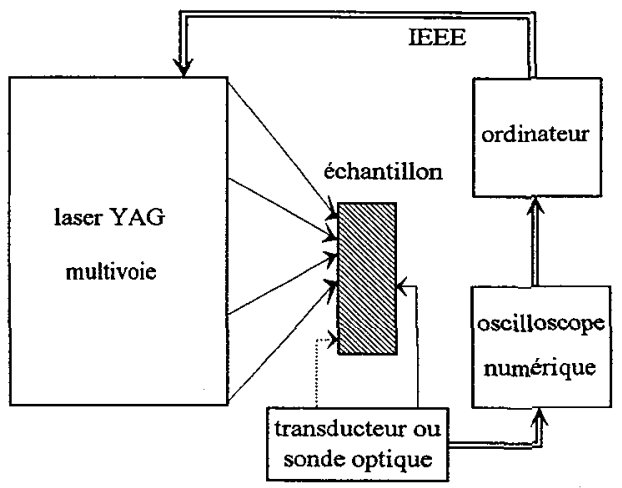

Fig. 2. Procédure expérimentale avec le laser multivoie en face de l'échantillon et le système de détection.

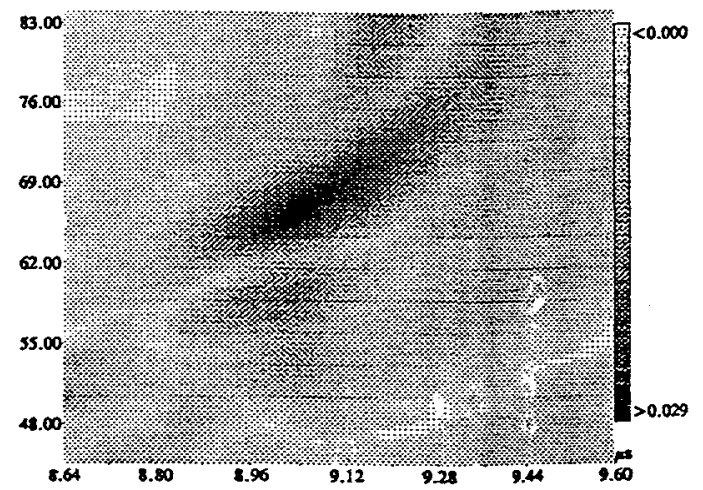

Fig. 4. B-Scan montrant la détection d'un trou $(\emptyset=1 \mathrm{~mm})$ situé à une distance de $15 \mathrm{~mm}$ sous la surface de l'échantillon dans la direction $\alpha=65^{\circ}$ par rapport au centre du réseau.

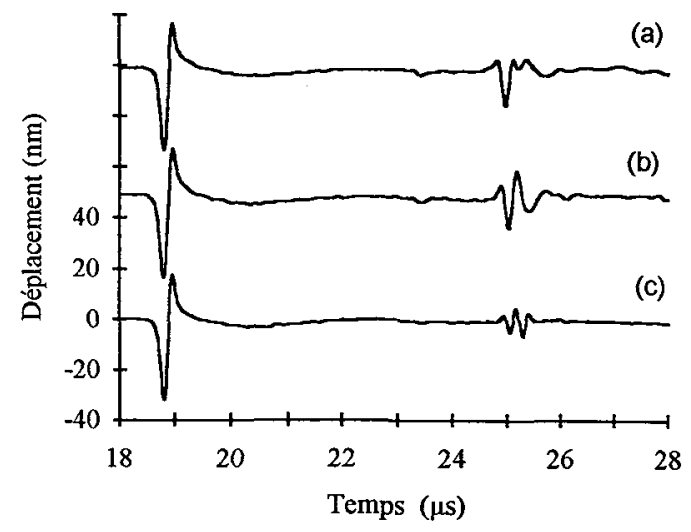

Fig. 6. Détection de fentes de largeur $0,2 \mathrm{~mm}$ de différentes profondeurs $h$ à la surface d'une plaque de duralumin. (a) $h=1 \mathrm{~mm}$, (b) $h=0,4 \mathrm{~mm}$, (c) $h=0,1 \mathrm{~mm}$. 